\title{
Confinement and Green functions in Landau-gauge QCD
}

\author{
Reinhard Alkofer* \\ Institut für Physik, Karl-Franzens-Universität, Universitätsplatz 5, A-8010 Graz, Austria \\ E-mail: reinhard.alkofer@uni-graz.at
}

\section{Christian S. Fischer}

Institut für Physik, TU Darmstadt, Schlossgartenstr. 9, D-64289 Darmstadt, Germany

\section{Markus Q. Huber}

Institut für Physik, Karl-Franzens-Universität, Universitätsplatz, 5, A-8010 Graz, Austria

\section{Felipe J. Llanes-Estrada}

Universidad Complutense de Madrid, Depto. Física Teórica I. 28040 Madrid, Spain

\section{Kai Schwenzer}

Institut für Physik, Karl-Franzens-Universität, Universitätsplatz, 5, A-8010 Graz, Austria

\begin{abstract}
In a functional approach to QCD the infrared behaviour of Landau gauge Green functions is investigated. Positivity violation for, and thus confinement of, gluons is demonstrated, and the analytic structure of the gluon propagator is studied. Quark confinement is related to an infrared divergence of the quark-gluon vertex. In the latter various components are dynamically induced due to the spontaneous breaking of chiral symmetry. As a result an infrared finite running coupling in the Yang-Mills sector is derived whereas the running coupling related to the quark-gluon vertex is infrared divergent. Based on a truncation for the quark-gluon vertex Dyson-Schwinger equation, which respects the analytically determined infrared behavior, numerical results for the coupled system of the quark propagator and vertex Dyson-Schwinger equations are presented. The resulting quark mass function as well as the vertex function show only a very weak dependence on the current quark mass in the deep infrared. From this we infer by an analysis of the quark-quark scattering kernel a linearly rising quark potential with an almost mass independent string tension in the case of broken chiral symmetry.
\end{abstract}

8th Conference Quark Confinement and the Hadron Spectrum

September 1-6, 2008

Mainz, Germany

\footnotetext{
${ }^{*}$ Speaker.
} 


\section{Infrared behaviour of Landau gauge Yang-Mills theory}

Confinement, the $U_{A}(1)$ anomaly, and dynamical chiral symmetry breaking are supposed to be properties of infrared QCD. Despite the progress achieved in our understanding of these phenomena the underlying mechanisms as well as possible interrelations between them are not yet uncovered. This talk aims at summarizing what we can learn from QCD Green functions in the Landau gauge about these topics.

Explaining quark confinement, and hereby especially extracting the linearly rising static quarkantiquark potential and relating its properties to QCD degrees of freedom, has been the main objective of many quite different studies. In ref. [1] some of these pictures where quark confinement is related to

- the condensation of chromomagnetic monopoles (e.g. [2, 3]),

- the percolation of center vortices(e.g. [4]),

- the $\mathrm{AdS}_{5}$ / QCD correspondence (e.g. [5]),

- the Gribov-Zwanziger scenario in Coulomb gauge (e.g. [6, 7]), or

- the infrared behaviour of Landau gauge Green functions $[8,9,10]$,

have been briefly reviewed. These explanations for confinement are seemingly different but there are surprising relations between them which are not yet understood. Given the current status one has to note that these pictures are definitely not mutually exclusive but simply reveal only different aspects of the confinement phenomenon. A similar statement on the relation between seemingly different scenarios is definitely true when considering the $U_{A}(1)$ anomaly, see $e$.g. the discussion in ref. [11]. This should be kept in mind when in the following the results based on investigations of Landau gauge Green functions are presented.

Employing Green functions dynamical chiral symmetry breaking reflects itself directly in terms of the quark propagator, see refs. $[8,9,12,13]$ and references therein. However, as we have demonstrated recently this picture is incomplete as it does not take into account the effects of dynamical chiral symmetry breaking on higher $n$-point functions $[14,15,16]$. This issue will be discussed in detail in this talk. To derive it one needs first some insight into the infrared behaviour of gluons and ghosts.

\subsection{Infrared exponents of gluons and ghosts}

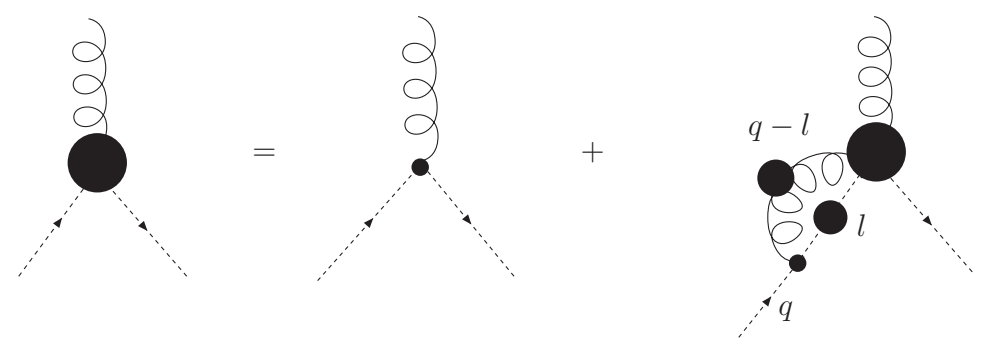

Figure 1: The Dyson-Schwinger equation for the ghost-gluon vertex.

The possibly best starting point for the investigation of the infrared behaviour of gluons and ghosts is given by the Dyson-Schwinger equation for the ghost-gluon vertex function, see fig. 1 . 
(NB: A MATHEMATICA package to derive Dyson-Schwinger equations can be found in [17].) In the Landau gauge the gluon propagator is transverse, and therefore one can employ the relation

$$
l_{\mu} D_{\mu v}(l-q)=q_{\mu} D_{\mu v}(l-q)
$$

to conclude that the ghost-gluon vertex stays finite when the outgoing ghost momentum vanishes, i.e. when $q_{\mu} \rightarrow 0$ [18]. This argument is valid to all orders in perturbation theory, a truely nonperturbative justification of the infrared finiteness of this vertex has been given in refs. [19, 20, 21].

Knowing this property of the ghost-gluon vertex the Dyson-Schwinger equation for the ghost propagator, see fig. 2, can be analysed. The only unknown functions in the deep infrared are the

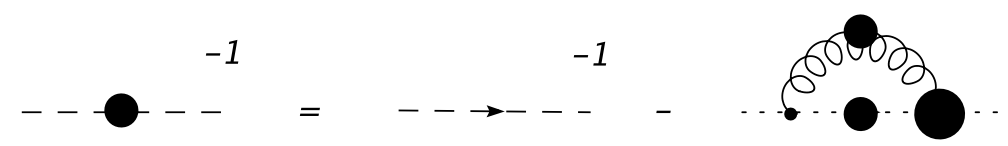

Figure 2: The Dyson-Schwinger equation for the ghost propagator.

gluon and the ghost propagators, parameterized in the Euclidean domain as

$$
D_{\mu v}(k)=\frac{Z\left(k^{2}\right)}{k^{2}}\left(\delta_{\mu v}-\frac{k_{\mu} k_{v}}{k^{2}}\right), \quad D_{G}(k)=-\frac{G\left(k^{2}\right)}{k^{2}} .
$$

In Landau gauge these propagators are best described by two invariant functions, $Z\left(k^{2}\right)$ and $G\left(k^{2}\right)$, respectively. As solutions of renormalized equations, these functions depend also on the renormalization scale $\mu$. Furthermore, assuming that QCD Green functions can be expanded in asymptotic series, the integral in the ghost Dyson-Schwinger equation can be split up in three pieces: an infrared integral, an ultraviolet integral, and an expression for the ghost wave function renormalization. Hereby it is the resulting equation for the latter quantity which allows one to extract definite information [22] without using any truncation or ansatz.

Recently it became clear that there are two distinct types of solutions, see ref. [23] and references therein. In this talk we will focus on the so-called scaling solution where the infrared behaviour of the gluon and ghost propagators is given by power laws. The corresponding exponents are uniquely related such that the gluon exponent is minus two times the ghost exponent [24]. As we will see later on this implies an infrared fixed point for the corresponding running coupling. The signs of the exponents are such that the gluon propagator is infrared suppressed as compared to the one for a free particle, the ghost propagator is infrared enhanced.

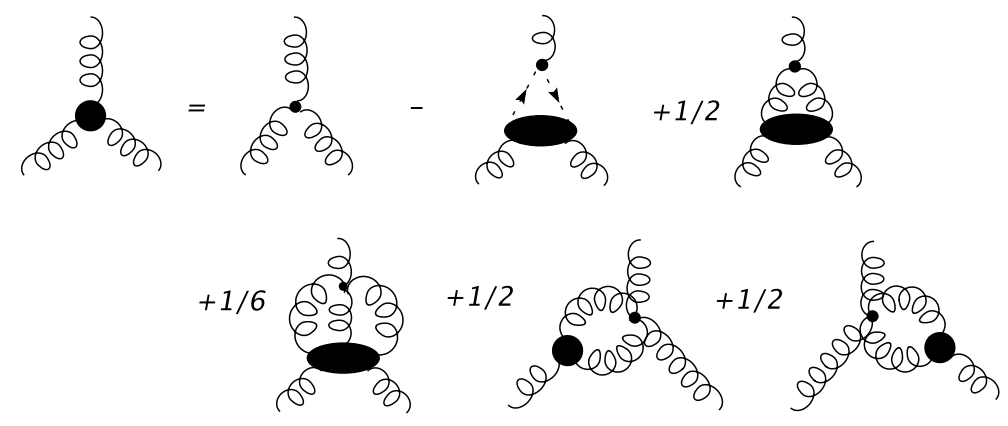

Figure 3: The Dyson-Schwinger equation for the 3-gluon vertex. 
Using this infrared power laws for the Yang-Mills propagators one can infer the infrared behaviour of higher $n$-point functions. To this end the $n$-point Dyson-Schwinger equations have been studied in a skeleton expansion, i.e. a loop expansion using dressed propagators and vertices. Furthermore, an asymptotic expansion has been applied to all primitively divergent Green functions [25]. As an example consider the Dyson-Schwinger equation for the 3-gluon vertex which is diagrammatically represented in fig. 3. Its skeleton expansion, see fig. 4 , can be constructed via the

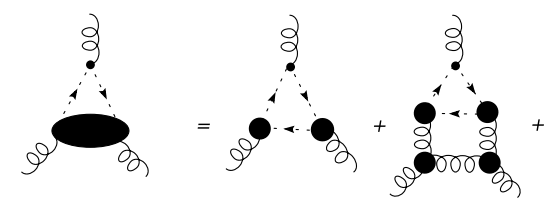

(a)

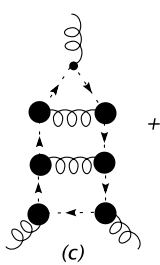

(c)

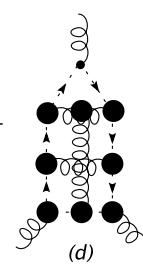

(d)

Figure 4: An example for the skeleton expansion of the 3-gluon vertex.

insertions given in fig. 5. These insertions have vanishing infrared anomalous dimensions which

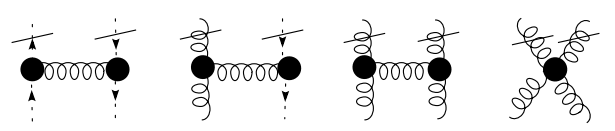

Figure 5: Insertions to reconstruct higher orders in the skeleton expansion.

implies that the resulting higher order terms feature the same infrared scaling. Based on this the following general infrared behaviour for one-particle irreducible Green functions with $2 n$ external ghost legs and $m$ external gluon legs can be derived [25, 26]:

$$
\Gamma^{n, m}\left(p^{2}\right) \sim\left(p^{2}\right)^{(n-m) \kappa+(1-n)(d / 2-2)}
$$

where $\kappa$ is one yet undetermined parameter, and $d$ is the space-time dimension.

Here two remarks are in order: First, exploiting Dyson-Schwinger and Exact Renormalization Group Equations one can show that this infrared solution is unique [27]. Second, there are additional divergences when only some of the momenta of the $n$-point functions are vanishing [28].

\subsection{Infrared fixed point of the running coupling in the Yang-Mills sector}

The infrared solutions (1.3) include

$$
G\left(p^{2}\right) \sim\left(p^{2}\right)^{-\kappa}, \quad Z\left(p^{2}\right) \sim\left(p^{2}\right)^{2 \kappa} \quad \Gamma^{3 g}\left(p^{2}\right) \sim\left(p^{2}\right)^{-3 \kappa}, \quad \Gamma^{4 g}\left(p^{2}\right) \sim\left(p^{2}\right)^{-4 \kappa} .
$$

This implies that the running couplings related to these vertex functions possess an infrared fixed point:

$$
\begin{aligned}
\alpha^{g h-g l}\left(p^{2}\right) & =\alpha_{\mu} G^{2}\left(p^{2}\right) Z\left(p^{2}\right) \sim \frac{\text { const }_{g h-g l}}{N_{c}}, \quad \alpha^{3 g}\left(p^{2}\right)=\alpha_{\mu}\left[\Gamma^{3 g}\left(p^{2}\right)\right]^{2} Z^{3}\left(p^{2}\right) \sim \frac{\text { const }_{3 g}}{N_{c}}, \\
\alpha^{4 g}\left(p^{2}\right) & =\alpha_{\mu} \Gamma^{4 g}\left(p^{2}\right) Z^{2}\left(p^{2}\right) \sim \frac{\text { const }_{4 g}}{N_{c}} .
\end{aligned}
$$

The infrared value of the coupling related to the ghost-gluon vertex can be computed to be $[19,29]$ :

$$
\alpha^{g h-g l}(0)=\frac{4 \pi}{6 N_{c}} \frac{\Gamma(3-2 \kappa) \Gamma(3+\kappa) \Gamma(1+\kappa)}{\Gamma^{2}(2-\kappa) \Gamma(2 \kappa)} .
$$

This yields $\alpha^{g h-g l}(0)=2.972$ for $N_{c}=3$ and $\kappa=(93-\sqrt{1201}) / 98 \simeq 0.595353$, which is the value obtained with a bare ghost-gluon vertex. 


\subsection{Gluon confinement by positivity violation for the gluon propagator}

Positivity violation for a propagator entails that the corresponding field is not related to an asymptotic state and thus to a particle. In the cases of gluons one can infer gluon confinement from such a scenario, see e.g. [30] and references therein. Therefore positivity violation of the propagator of transverse gluons has been for a long time a conjecture which has been convincingly verified by now, [31, 32] as well as references therein. The basic feature is hereby the infrared suppression of transverse gluons caused by the infrared enhancement of ghosts. Being related to the confinement of tranverse gluons [30] it is certainly worth to have a closer look at the underlying analytic structure of the gluon propagator.

As the infrared exponent $\kappa$ in the infrared power laws is an irrational number this implies already that the gluon propagator possesses a cut on the negative real $p^{2}$ axis. It is possible to fit the solution for the gluon propagator accurately without introducing further singularities in the complex $p^{2}$ plane [31]:

$$
Z_{\mathrm{fit}}\left(p^{2}\right)=w\left(\frac{p^{2}}{\Lambda_{\mathrm{QCD}}^{2}+p^{2}}\right)^{2 \kappa}\left(\alpha_{\mathrm{fit}}\left(p^{2}\right)\right)^{-\gamma}
$$

Hereby $w$ is a normalization parameter, and $\gamma=\left(-13 N_{c}+4 N_{f}\right) /\left(22 N_{c}-4 N_{f}\right)$ is the one-loop value for the anomalous dimension of the gluon propagator. The running coupling is expressed as [34]:

$$
\alpha_{\mathrm{fit}}\left(p^{2}\right)=\frac{\alpha_{S}(0)}{1+p^{2} / \Lambda_{\mathrm{QCD}}^{2}}+\frac{4 \pi}{\beta_{0}} \frac{p^{2}}{\Lambda_{\mathrm{QCD}}^{2}+p^{2}}\left(\frac{1}{\ln \left(p^{2} / \Lambda_{\mathrm{QCD}}^{2}\right)}-\frac{1}{p^{2} / \Lambda_{\mathrm{QCD}}^{2}-1}\right)
$$

with $\beta_{0}=\left(11 N_{c}-2 N_{f}\right) / 3$. Note that the gluon propagator (1.7) possesses an important property: Wick rotation is directly possible!

\subsection{Summary on Yang-Mills sector}

To summarize this section we note that the here discussed scaling solution for Green functions in an Yang-Mills theory implies that the gluon propagator vanishes on the light cone, and $n$-point gluon vertex functions diverge on the light cone. Therefore an attempt to kick a gluon free, i.e. to produce a real gluon, immediately results in production of infinitely many virtual soft gluons to produce perfect color charge screening. Positivity violation (which implies BRST quartet cancelation [30]) guarantees that this screening is total, or phrased otherwise, that gluons are confined.

\section{Dynamically induced scalar quark confinement}

\subsection{Self-consistency between the quark propagator and the quark-gluon vertex}

As detailed above Landau gauge Green functions provide a consistent picture for gluon confinement. However, due to the infrared suppression of the gluon propagator quark confinement seems even more unexplainable. To proceed it turns out to be necessary to study the DysonSchwinger equation for the quark propagator together with the one for the quark-gluon vertex $[8,9,10,16,33,34]$. Therefore a detailed study of this three-point function, and especially its 


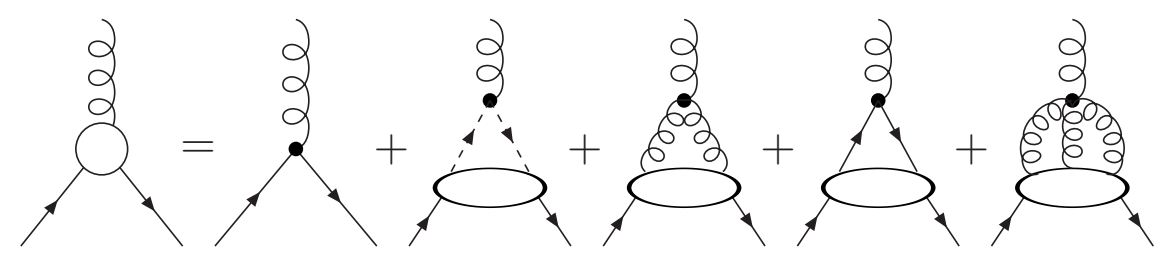

Figure 6: The Dyson-Schwinger equation for the quark-gluon vertex.

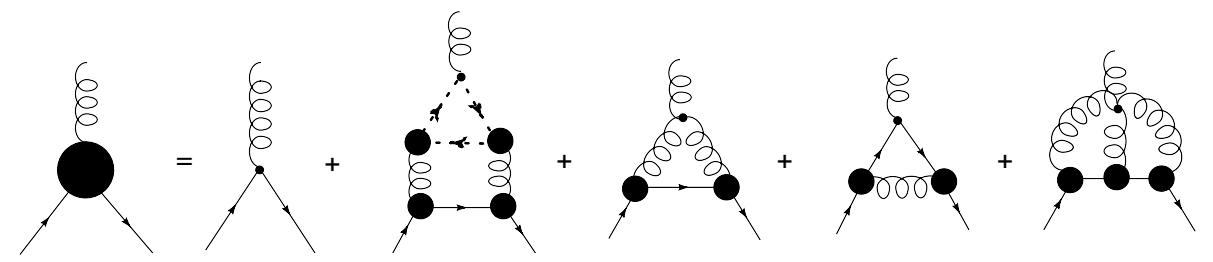

Figure 7: The skeleton expansion for the quark-gluon vertex.

infrared behaviour, is mandatory. Its Dyson-Schwinger equation is diagrammatically depicted in fig. 6, its skeleton expansion in fig. 7. Hereby a drastic difference of the quarks as compared to Yang-Mills fields has to be taken into account: They possess a mass, and in addition one has to take into account that dynamical chiral symmetry breaking and thus dynamical mass generation may (and will) occur.

The infrared analysis of the Yang-Mills theory has been generalized to full, although quenched, QCD in ref. [16]. Let us first consider a limit where the masses of the valence quarks are large, i.e. $m>\Lambda_{\mathrm{QCD}}$. The remaining scales below $\Lambda_{\mathrm{QCD}}$ are those of the external momenta of the propagators and vertex functions. The Dyson-Schwinger equations are then employed to determine the selfconsistent solutions in terms of powers of the small external momentum scale $p^{2} \ll \Lambda_{\mathrm{QCD}}$. The equations which have to be considered in addition to the ones of Yang-Mills theory are the ones for the quark propagator and the quark-gluon vertex which in turn have to be solved self-consistently.

\subsection{The quark-gluon vertex, chiral symmetry, and quark confinement}

The fully renormalized quark-gluon vertex $\Gamma_{\mu}$ consists of up to twelve linearly independent Dirac tensors. Half of these vanish in case chiral symmetry would be realized in the Wigner-Weyl mode, i.e. these tensor structures can only be non-vanishing either if chiral symmetry is explicitely broken by current masses and/or chiral symmetry is realized in Nambu-Goldstone mode (i.e. spontaneously broken). From a solution of the Dyson-Schwinger equations we infer that these "scalar" structures are, in the chiral limit, generated non-perturbatively together with the dynamical quark mass function in a self-consistent fashion. Thus dynamical chiral symmetry breaking reflects itself not only in the propagator but also in the quark-gluon vertex.

Performing an infrared analysis one obtains an infrared divergent solution for the quark-gluon vertex such that Dirac vector and "scalar" components of this vertex are infrared divergent with exponent $-\kappa-\frac{1}{2}$ when either all momenta or when only the gluon momenta vanish $[14,16]$. A numerical solution of a truncated set of Dyson-Schwinger equations confirms this infrared behavior, see fig. 8. The driving pieces of this solution are the scalar Dirac amplitudes of the quark-gluon vertex and the scalar part of the quark propagator. Both pieces are only present when chiral symmetry is broken, either explicitely or dynamically. As can be seen from fig. 8 the function $\lambda_{1}$ multiplying 
the tree-level tensor structure $\gamma_{\mu}$ is only the leading one in the ultraviolet, in the infrared the scalar component $\lambda_{2}$ is even larger than the vector component $\lambda_{1}$.

\section{b-quark}

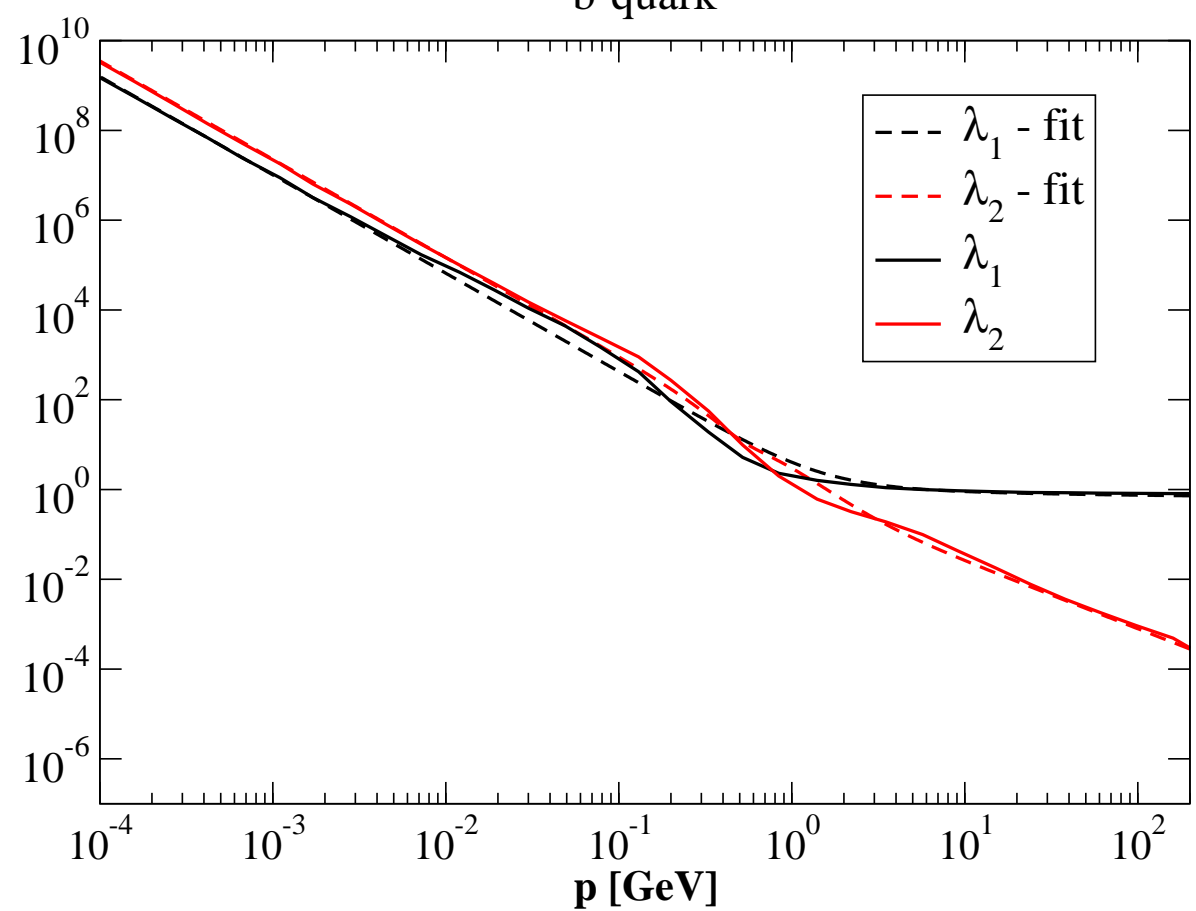

Figure 8: The leading vector component $\lambda_{1}$ and a scalar component $\lambda_{2}$ of the quark-gluon vertex for a current mass corresponding to a $b$ quark. Adapted from ref. [16]

The quark-gluon vertex may also serve to extract a running coupling. Using

$$
\Gamma^{q g}\left(p^{2}\right) \sim\left(p^{2}\right)^{-1 / 2-\kappa}, Z_{f}\left(p^{2}\right) \sim \text { const }, Z\left(p^{2}\right) \sim\left(p^{2}\right)^{2 \kappa}
$$

one obtains

$$
\alpha^{q g}\left(p^{2}\right)=\alpha_{\mu}\left[\Gamma^{q g}\left(p^{2}\right)\right]^{2}\left[Z_{f}\left(p^{2}\right)\right]^{2} Z\left(p^{2}\right) \sim \frac{\text { const }_{q g}}{N_{c}} \frac{1}{p^{2}},
$$

and thus a coupling which is singular in the infrared contrary to the couplings from the Yang-Mills vertices.

To determine whether this already relates to quark confinement the anomalous infrared exponent of the four-quark function is determined. The static quark potential can be obtained from this four-quark one-particle irreducible Green function, which, including the canonical dimensions, behaves like $\left(p^{2}\right)^{-2}$ for $p^{2} \rightarrow 0$ due to the singularity of the quark-gluon vertex for vanishing gluon momentum. Using a well-known relation for a function $F \propto\left(p^{2}\right)^{-2}$ yields

$$
V(\mathbf{r})=\int \frac{d^{3} p}{(2 \pi)^{3}} F\left(p^{0}=0, \mathbf{p}\right) e^{i \mathbf{p r}} \sim|\mathbf{r}|
$$

for the static quark-antiquark potential $V(\mathbf{r})$. At this point one notes that, given the infrared divergence of the quark-gluon vertex as found in the scaling solution of the coupled system of DysonSchwinger equations, the vertex overcompensates the infared suppression of the gluon propagator 
such that one obtains a linearly rising potential. The surprising fact is that this potential is dynamically induced and in the infrared dominantly scalar.

To provide further understanding for the here found relation between chiral symmetry breaking and quark confinement one may keep chiral symmetry artificially in Wigner-Weyl mode, i.e. in the chiral limit one forces the quark mass term as well as the "scalar" terms in the quark-gluon vertex to be zero. The result of such a procedure is that then the running coupling from the quark-gluon vertex is no longer diverging but goes to a fixed point in the infrared similar to the couplings from the Yang-Mills vertices. Correspondingly, one obtains a $1 / r$ behaviour of the static quark potential. The "enforced" restoration of chiral symmetry is therefore directly linked with the disappearance of quark confinement. The infared properties of the quark-gluon vertex in the "unforced" solution thus constitute a novel mechanism that directly links chiral symmetry breaking with quark confinement.

\subsection{Mass dependence}

As mentioned above, based on a truncation for the quark-gluon vertex Dyson-Schwinger equation which respects the analytically determined infrared behavior, numerical results for the coupled system of the quark propagator and vertex Dyson-Schwinger equations have been obtained [16]. In fig. 9 the resulting quark mass function is presented for the values of current masses related to $u / d, s, c$ and $b$ quarks. These quark mass functions as well as the vertex functions show only a very

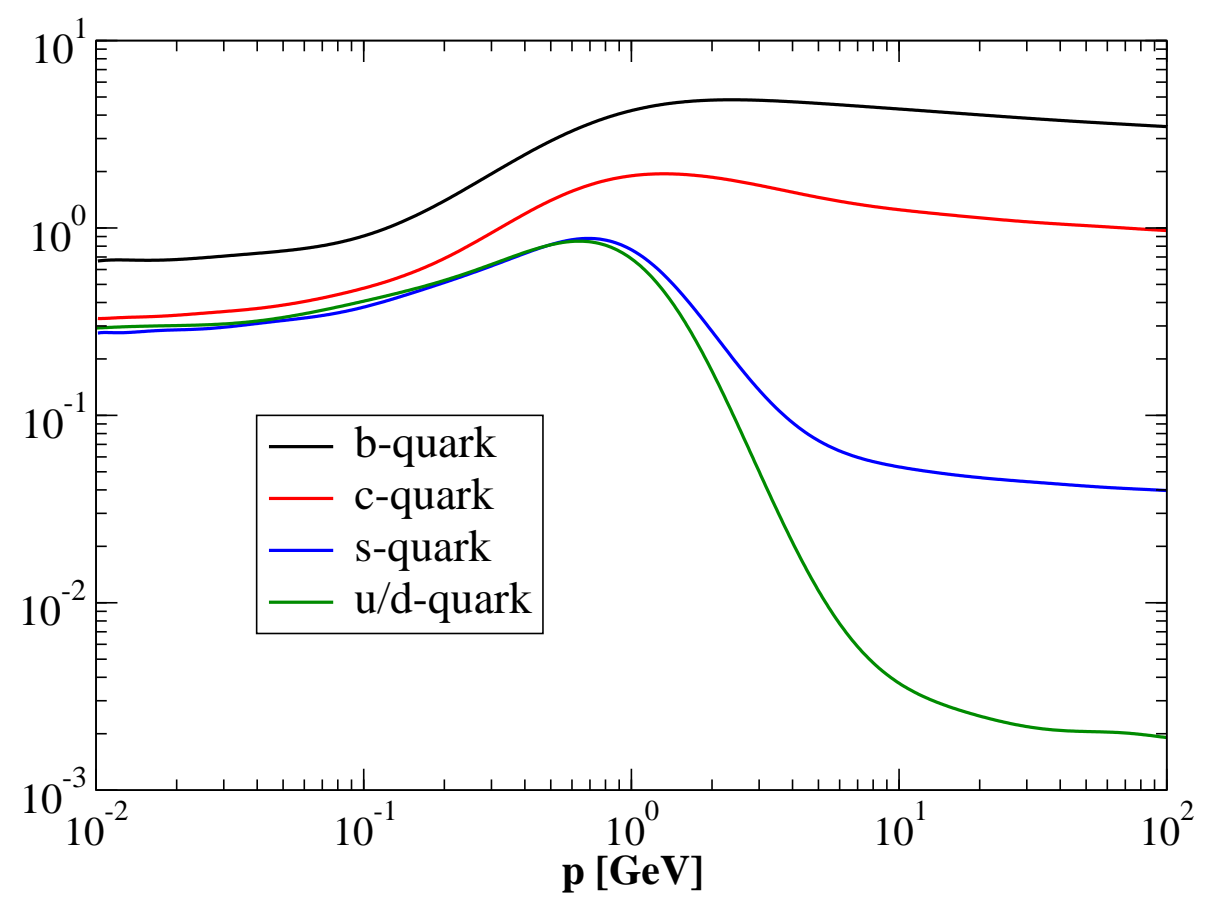

Figure 9: The quark mass functions for several values of current masses. Adapted from ref. [16]

weak dependence on the current quark mass in the deep infrared. First of all, the infrared exponent for all vertex functions are mass independent. Second, comparing the coefficients of these power laws, given in table 1, we see a much smaller dependence on the quark mass than could have been anticipated from a naive analysis. As the static potential will be build up by the infrared divergent parts of the quark-gluon vertex we thus predict that the string tension is almost mass-independent. 


\begin{tabular}{c|c|c|c|c|} 
& $u / d$ & $s$ & $c$ & $b$ \\
\hline$m\left(\mu^{2}\right)[\mathrm{MeV}]$ & 3 & 50 & 1200 & 4200 \\
\hline$M(0)[\mathrm{MeV}]$ & 270 & 270 & 320 & 650 \\
\hline$\lambda_{1}\left[\mathrm{GeV}^{1 / 2+\kappa}\right]$ & 3.95 & 3.60 & 2.00 & 2.73 \\
\hline$\lambda_{2}\left[\mathrm{GeV}^{1 / 2+\kappa}\right]$ & 8.70 & 7.97 & 4.44 & 6.08
\end{tabular}

Table 1: The infrared coefficients for the dressing functions $\lambda_{1,2}$ for different current quark masses $m\left(\mu^{2}\right)$ (at the renormalization point $\left.\mu^{2}=170 \mathrm{GeV}\right)$. Also given are the values of the mass function at zero momentum.

Provided the Coulombic part of potential has still a large effect in the bottomonium spectrum this is in agreement with experimental splittings of charmonia and bottonia systems.

Thus our analysis provides (at least) two surprises: First, the longstanding discussion on whether confinement is of a vector or of a scalar nature is oversimplified. Dynamically generated structures of vector, scalar and tensor types in the quark-gluon vertex lead to a rich structure of the quark-antiquark interaction. Second, the mass dependence of the string tension does not seem to follow any easy rule, $c f$. also recent improved lattice data [35]. These and other details of the confining interaction clearly deserve more investigations.

\subsection{Summary and outlook on quark sector}

The scaling solution for Landau-gauge QCD Green functions has far reaching consequences for the infrared behaviour of quarks. The resulting dynamical chiral symmetry breaking and the related dynamical mass generation lead to an infrared trivial quark propagator. But dynamical chiral symmetry breaking also occurs in the quark-gluon vertex. Hereby several components, and especially also the "scalar" ones, diverge on the quark "mass" shell.

An attempt to kick a quark free, i.e. to produce a real quark, immediately results in production of infinitely many virtual soft gluons. Hereby these gluons do not only couple vector-like but also scalar-like! And the vertex function diverges such that effectively a linearly rising potential is produced. One obtains therefore infrared slavery and quark confinement.

Although these results are in itself quite surprising and encouraging they provide only the starting point for an understanding of quark confinement in functional approaches. Questions which will be investigated further include:

- How does the formation of a string between colour sources reflect itself in Green functions?

- Can we learn about the properties of the confining field configurations from Green functions?

- How does all this relate to other (partially) successful approaches to understand the phenomenon of quark confinement?

\section{Acknowledgements}

R.A. thanks the organisers of Confinement 8 for all their efforts which made this extraordinary conference possible. We are grateful to A. Cucchieri, A. Maas, T. Mendez, J. Pawlowski, and L. v. Smekal for interesting discussions.

R.A. was supported by the FWF grant P20592-N16, C.S. F. the Helmholtz-University Young Investigator Grant No. VH-NG-332, and K. S. by the FWF Lise-Meitner grant M979-N16. 


\section{References}

[1] R. Alkofer and J. Greensite, J. Phys. G34 (2007) S3 [arXiv:hep-ph/0610365].

[2] S. Mandelstam, Phys. Rept. 23 (1976) 245.

[3] A. Di Giacomo, B. Lucini, L. Montesi and G. Paffuti, Phys. Rev. D 61 (2000) 034503 [arXiv:hep-lat/9906024];

[4] J. Greensite, Prog. Part. Nucl. Phys. 51 (2003) 1 [arXiv:hep-lat/0301023].

[5] J. M. Maldacena, Phys. Rev. Lett. 80, 4859 (1998) [arXiv:hep-th/9803002].

[6] V. Gribov, Nucl. Phys. B139 (1978) 1.

[7] D. Zwanziger: Nucl. Phys. B518 (1998) 237.

[8] R. Alkofer and L. von Smekal, Phys. Rept. 353 (2001) 281 [arXiv:hep-ph/0007355].

[9] C. S. Fischer, J. Phys. G: Nucl. Part. Phys. 32 (2006) R253 [arXiv:hep-ph/0605173].

[10] R. Alkofer, Braz. J. Phys. 37 (2007) 144 [arXiv:hep-ph/0611090].

[11] R. Alkofer, C. S. Fischer and R. Williams, Eur. Phys. J. A 38 (2008) 53 [arXiv:0804.3478 [hep-ph]].

[12] V. A. Miransky, Phys. Lett. B 165, 401 (1985).

[13] M. R. Pennington, in Proceedings of the Workshop on Nonperturbative Methods in Quantum Field Theory, edited by A. W. Schreiber, A. G. Williams, and A. W. Thomas, p. 49, Adelaide, 1998, World Scientific; arXiv:hep-th/9806200.

[14] R. Alkofer, C. S. Fischer and F. J. Llanes-Estrada, Mod. Phys. Lett. A23 (2008) 1105 [arXiv:hep-ph/0607293].

[15] R. Alkofer, C. S. Fischer, F. J. Llanes-Estrada and K. Schwenzer, PoS LAT2007 (2007) 286 [arXiv:0710.1154 [hep-ph]].

[16] R. Alkofer, C. S. Fischer, F. J. Llanes-Estrada and K. Schwenzer, Ann. Phys., in print [arXiv:0804.3042 [hep-ph]].

[17] R. Alkofer, M. Q. Huber and K. Schwenzer, Comp. Phys. Comm., in print [arXiv:0808.2939 [hep-th]].

[18] J. C. Taylor, Nucl. Phys. B33 (1971) 436.

[19] C. Lerche and L. von Smekal, Phys. Rev. D 65 (2002) 125006 [arXiv:hep-ph/0202194].

[20] A. Cucchieri, T. Mendes, and A. Mihara JHEP 12 (2004) 012 [arXiv:hep-lat/0408034].

[21] W. Schleifenbaum, A. Maas, J. Wambach, and R. Alkofer, Phys. Rev. D72 (2005) 014017 [arXiv:hep-ph/0411052].

[22] P. Watson and R. Alkofer, Phys. Rev. Lett. 86 (2001) 5239 [arXiv:hep-ph/0102332].

[23] C. S. Fischer, A. Maas, and J. M. Pawlowski, arXiv:0810.1987 [hep-ph]

[24] L. von Smekal, R. Alkofer and A. Hauck, Phys. Rev. Lett. 79 (1997) 3591 [arXiv:hep-ph/9705242];

L. von Smekal, A. Hauck and R. Alkofer, Annals Phys. 267 (1998) 1 [arXiv:hep-ph/9707327].

[25] R. Alkofer, C. S. Fischer and F. J. Llanes-Estrada, Phys. Lett. B 611 (2005) 279 [arXiv:hep-th/0412330]; R. Alkofer et al., arXiv:nucl-th/0601032.

[26] M. Q. Huber, R. Alkofer, C. S. Fischer and K. Schwenzer, Phys. Lett. B 659 (2008) 434 [arXiv:0705.3809 [hep-ph]]; R. Alkofer, C. S. Fischer, M. Q. Huber and K. Schwenzer, PoS LAT2007 (2007) 329 [arXiv:0710.1054 [hep-ph]].

[27] C. S. Fischer and J. M. Pawlowski, Phys. Rev. D 75 (2007) 025012 [arXiv:hep-th/0609009].

[28] R. Alkofer, M. Q. Huber and K. Schwenzer, arXiv:0801.2762 [hep-th].

[29] C. S. Fischer and R. Alkofer, Phys. Lett. B536 (2002) 177 [arXiv:hep-ph/0202202]; C. S. Fischer, R. Alkofer and H. Reinhardt, Phys. Rev. D65 2002094008 [arXiv:hep-ph/0202195]; R. Alkofer, C. S. Fischer and L. von Smekal, Acta Phys. Slov. 52(2002) 191 [arXiv:hep-ph/0205125].

[30] L. von Smekal and R. Alkofer, arXiv:hep-ph/0009219.

[31] R. Alkofer, W. Detmold, C. S. Fischer and P. Maris, Phys. Rev. D 70 (2004) 014014 [arXiv:hep-ph/0309077]; Nucl. Phys. Proc. Suppl. 141 (2005) 122.

[32] P. O. Bowman et al., Phys. Rev. D 76 (2007) 094505 [arXiv:hep-lat/0703022].

[33] K. Schwenzer, arXiv:0811.3608 [hep-ph].

[34] C. S. Fischer and R. Alkofer, Phys. Rev. D 67 (2003) 094020 [arXiv:hep-ph/0301094].

[35] Y. Koma, M. Koma and H. Wittig, arXiv:0711.2322 [hep-lat]. 